\title{
Green and solvent-free procedure for microwave-assisted synthesis of 2,4,6-triarylpyridines catalysed using $\mathrm{MgAl}_{2} \mathrm{O}_{4}$ nanocrystals
}

\author{
JAVAD SAFARI*, SOHEILA GANDOMI-RAVANDI and MAHMOUD BORJIAN BORUJENI \\ Laboratory of Organic Chemistry Research, Department of Chemistry, College of Chemistry, \\ University of Kashan, Kashan, P.O. Box: 87317-51167, Kashan, Islamic Republic of Iran \\ e-mail: Safari@kashanu.ac.ir
}

MS received 18 March 2013; revised 20 May 2013; accepted 18 June 2013

\begin{abstract}
Design and development of a heterogeneous nanocatalyst for condensation reaction of acetophenone derivatives, aromatic aldehydes, and ammonium acetate to yield 2,4,6-triarylpyridines followed by microwave irradiation is described. Nanocrystalline $\mathrm{MgAl}_{2} \mathrm{O}_{4}$ as a novel heterogeneous recyclable catalyst shows high activity for the above reaction. In addition, the easily recoverable nanosized catalysts showed good reusability.
\end{abstract}

Keywords. Triarylpyridine; microwave irradiation; multi-component reaction; heterogeneous catalyst.

\section{Introduction}

Microwave technology is recognized as a very important tool in organic synthesis and therefore microwave chemistry can provide access to synthetic transformations. ${ }^{1}$ Microwave heating is observed to yield a number of unique advantages including non-contact and uniform heating, rapid and selective heating, volumetric heating, reduced processing costs, quick start-up and stopping. ${ }^{2}$ Furthermore, reactions under microwave have the great advantage of not using organic solvents (solvent-free), and thus such reactions are more environment-friendly and generate less waste. It has been well-established that microwaves shorten reaction time and improve product yield without the use of solvents. ${ }^{3-5} \mathrm{~N}$-heterocyclic compounds, such as pyridines have a unique position in synthetic chemistry. The pyridyl heterocyclic nucleus is a building block in molecules of natural products, pharmaceuticals such as vasodilator, anticonvulsant, antiepileptic, anaesthetic and agrochemicals such as pesticides and herbicidals. ${ }^{6-9}$ Owing to their $\pi$-stacking ability, directional $\mathrm{H}$-bonding and coordination, triarylpyridines are widely used in supramolecular chemistry. ${ }^{10}$ Previously, 2,4,6-triarylpyridines (Krohnke pyridine) were prepared through the reaction of $N$-phenacylpyridinium salts with $\alpha, \beta$-unsaturated ketones in the presence of $\mathrm{NH}_{4} \mathrm{OAc},{ }^{11,12}$ the condensation of 1,5diketones with formamide-ammonium formate ${ }^{13}$ and by other synthetic procedures including the Chichibabin

*For correspondence method. ${ }^{14-17}$ More recently, many improved methods and procedures for preparation of 2,4,6-triarylpyridines have been reported such as reaction of $\alpha$-ketoketene dithioacetals with methyl ketones, ${ }^{18}$ reaction of $N$ phosphinylethanimines with aldehydes, ${ }^{19}$ reaction of chalcones with ammonium acetate, ${ }^{20}$ addition of lithiated $\beta$-enaminophosphonates to chalcones, ${ }^{21}$ reaction of chalcones and compounds possessing terminal $-\mathrm{CXNH}_{2}$ functionality, ${ }^{22,23}$ reaction of acetophenone and chalcone with a source of ammonia (urea, ammonium acetate, and guanidine), ${ }^{24-26}$ and condensation of acetophenones, benzaldehydes and $\mathrm{NH}_{4} \mathrm{OAc} .{ }^{27}$ Among all these methods, one-pot condensation reaction of acetophenones with aryl aldehydes, and ammonium acetate is a well-established protocol for the synthesis of 2,4,6-triarylpyridines. This one-pot condensation has been developed in the presence of catalytic amount of acetic acid, ${ }^{20} \mathrm{NaOH}$ in PEG-400, ${ }^{28}$ Preyssler type heteropolyacid $\mathrm{H}_{14}\left[\mathrm{NaP}_{5} \mathrm{~W}_{30} \mathrm{O}_{110}\right],{ }^{29}$ Bronsted acidic ionic liquid, ${ }^{30} \mathrm{HClO}_{4}-\mathrm{SiO}_{2},{ }^{31}$ cyanuric chloride, ${ }^{32} \mathrm{I}_{2},{ }^{33}$ wet 2,4,6-trichloro-1,3,5-triazine (TCT), ${ }^{32}$ pentafluorophenylammonium triflate (PFPAT), ${ }^{34} \mathrm{Bi}(\mathrm{OTf})_{3},{ }^{35}$ $\mathrm{H}_{2} \mathrm{SO}_{4}-\mathrm{SiO}_{2},{ }^{36}$ tetrabutylammonium hydrogen sulphate (TBAHS), ${ }^{37} \mathrm{PPA}-\mathrm{SiO}_{2},{ }^{38}$ and $\mathrm{N}$-bromosuccinimide or trichloroisocyanuric acid. ${ }^{39}$ In recent years, nano materials have emerged as powerful catalysts in various organic transformations such as $\mathrm{ZnO}$ nanoparticles, ${ }^{40}$ barium chloride dispersed on silica gel nanoparticles $^{41}$ for synthesis of 2,4,6-triarylpyridines, and using silica nanoparticles for preparation of highly substituted pyridines. ${ }^{42}$ The nanosized spinel particles have many applications because of their good physical and 


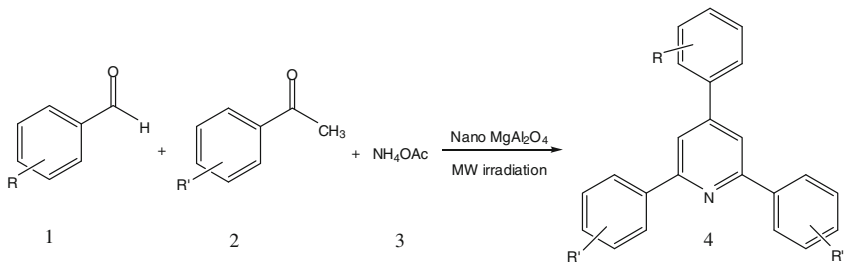

Scheme 1. Microwave-mediated synthesis of 2,4,6triarylpyridines in the presence of nanocrystalline $\mathrm{MgAl}_{2} \mathrm{O}_{4}$.

chemical properties. ${ }^{43}$ Magnesium aluminate spinel $\left(\mathrm{MgAl}_{2} \mathrm{O}_{4}\right)$ is one of the best known and widely used materials. It has good thermal shock resistance and high chemical inertness in both basic and acidic environments. ${ }^{44,45}$ Moreover, spinel has found application in catalyst support, ${ }^{46}$ dentistry, humidity sensors ${ }^{47}$ and ceramic pigments. ${ }^{48}$ However, to the best of our knowledge, the use of $\mathrm{MgAl}_{2} \mathrm{O}_{4}$ as catalyst in the synthesis of 2,4,6-triarylpyridines has not been reported. Herein, we introduce a simple, easy and one-pot synthesis for 2,4,6-triarylpyridines using nanocrystalline $\mathrm{MgAl}_{2} \mathrm{O}_{4}$ as a novel heterogeneous catalyst under microwave conditions (Scheme 1).

\section{Experimental}

\subsection{Materials and instruments}

All materials were purchased from Merck and Fluka in commercial grade. All yields refer to isolated products. Melting points were recorded on an electrothermal 9200 MK3 melting point. The FT-IR spectra were obtained on a Nicolet Magna 550 Fourier transform infrared (FT-IR) spectrophotometer as $\mathrm{KBr}$ disk. Elemental analyses were determined by using a Perkin-Elmer 2400 CHNS/O analyser instrument. Chemical ionization mass spectral data were obtained using a Micro Mass UK LTD mass spectrometer. The ${ }^{1} \mathrm{H}$ and ${ }^{13} \mathrm{C}$ Nuclear Magnetic Resonance (NMR) spectra $(400 \mathrm{MHz}, 100 \mathrm{MHz})$ were recorded on a Bruker Avance-DRX-400 spectrometer using Dimethyl sulfoxide (DMSO- $d_{6}$ ) as solvent and Tetramethylsilan (TMS) as an internal standard. The CEM-Discover Focused Monomode Microwave reactor $(2450 \mathrm{MHz}, 300 \mathrm{~W})$ was used for microwave irradiation. Microwave-mediated reactions were performed in sealed process vials under air with magnetic stirring.

\section{1a General method for synthesis of nano crystalline} $\mathrm{MgAl}_{2} \mathrm{O}_{4}: \quad \mathrm{Al}\left(\mathrm{NO}_{3}\right)_{3} \cdot 9 \mathrm{H}_{2} \mathrm{O}$ and $\mathrm{Mg}\left(\mathrm{NO}_{3}\right)_{2} \cdot 6 \mathrm{H}_{2} \mathrm{O}$ were used as starting materials. Stoichiometric amounts of magnesium and aluminum nitrates were added to well-stirred deionized water. Ethylene diamine was added dropwise to the well-stirred slurry for adjusting $\mathrm{pH}$ value to around 9. After precipitation, the slurry was stirred for another $30 \mathrm{~min}$ and then stirred at $80^{\circ} \mathrm{C}$ for $24 \mathrm{~h}$ under reflux conditions. Finally, the product was filtered and dried at $100^{\circ} \mathrm{C}$ for $24 \mathrm{~h}$ under flowing air and calcined at $700^{\circ}$ and $800^{\circ} \mathrm{C}$ for $5 \mathrm{~h}$. This method was obtained from literature. ${ }^{20}$

2.1b General method for one-pot synthesis of 2,4,6triarylpyridines under microwave irradiation: Aromatic aldehydes $(1 \mathrm{mmol})$, acetophenones $(2 \mathrm{mmol})$, $\mathrm{NH}_{4} \mathrm{OAc}$ (4 mmol), and nanocrystalline $\mathrm{MgAl}_{2} \mathrm{O}_{4}$ $(5 \mathrm{~mol} \%)$ were heated in a microwave oven for an appropriate time. Upon completion, hot ethanol was added to the mixture and the insoluble catalyst was filtered off. The pure products were obtained by recrystallization from $95 \%$ ethanol. The structures of the products $4 \mathbf{a}-\mathbf{q}$ were deduced from their IR, ${ }^{1} \mathrm{H}$ and ${ }^{13} \mathrm{C}$ NMR, MS and CHN analysis spectra.

\subsection{Spectral data}

2.2a 2,4,6-Triphenylpyridine $\left(\mathrm{C}_{23} \mathrm{H}_{17} \mathrm{~N}\right.$ 4a): White solid; m.p. $135-137^{\circ} \mathrm{C}(85 \%) ; R_{\mathrm{f}}$ (petroleum ether: ethylacetate): 7:3 (v/v) $=0.80 ; \mathrm{UV}\left(\mathrm{CHCl}_{3}\right) \lambda_{\max }=$ $304 \mathrm{~nm}$; FT-IR $(\mathrm{KBr})=3069,1597,1552,1494$, 1440, 1398, 1178, 1074, 1027, 867, 759, $692 \mathrm{~cm}^{-1}$; ${ }^{1} \mathrm{H}$ NMR $\left(400 \mathrm{MHz}, \mathrm{DMSO}-d_{6}\right) \delta 8.33-8.37(\mathrm{~d}, 4 \mathrm{H}$, ArH), 8.20 (s, 2H, ArH), 8.02-8.07 (d, 2H, ArH), 7.50-7.60 (m, 9H, ArH); ${ }^{13} \mathrm{C}$ NMR (100 MHz DMSO$\left.d_{6}\right): \delta 157.02,150.06,139.26,139.19,129.73,129.68$, 128.72, 128.39, 127.75, 127.4, 117.02; Mass (ES-MS): $m / z$ 308.1 $\left(\mathrm{M}^{+}\right)$; Anal. Calcd. for $\mathrm{C}_{23} \mathrm{H}_{17} \mathrm{~N}$ : C 89.87, $\mathrm{H}$ 5.57, N 4.56; found: C 89.54, H 5.42, N 4.67\%.

2.2b 4-(4-Chlorophenyl)-2,6-diphenylpyridine $\left(\mathrm{C}_{23} \mathrm{H}_{16} \mathrm{ClN} 4 \boldsymbol{b}\right)$ : White solid; m.p. $127-128^{\circ} \mathrm{C}(87 \%)$; $R_{\mathrm{f}}$ (petroleum ether:ethylacetate): $7: 3(\mathrm{v} / \mathrm{v})=0.83$; $\mathrm{UV}\left(\mathrm{CHCl}_{3}\right) \lambda_{\max }=304 \mathrm{~nm}$; FT-IR $(\mathrm{KBr})=3061$, 1599, 1543, 1489, 1449, 1414, 1384, 1237, 1090, 1013, $825,773,692 \mathrm{~cm}^{-1} ;{ }^{1} \mathrm{H}$ NMR (400 MHz, DMSO- $d_{6}$ ) $\delta$ 8.33-8.38 (d, $4 \mathrm{H}, J=7.2 \mathrm{~Hz}, \operatorname{ArH}), 8.14(\mathrm{~s}, 2 \mathrm{H}$, ArH), 7.78 (d, 2H, $J=8 \mathrm{~Hz}, \operatorname{ArH}), 7.62-7.650$ (d, 2H, $J=6.8 \mathrm{~Hz}, \mathrm{ArH}), 7.53-7.56(\mathrm{~m}, 6 \mathrm{H}, J=8 \mathrm{~Hz}, \mathrm{ArH})$; ${ }^{13} \mathrm{C}$ NMR $\left(100 \mathrm{MHz}\right.$ DMSO- $\left.d_{6}\right): \delta 157.05,148.64$, 139.18, 136.94, 134.7, 129.64, 129.47, 129.44, 129.17, 116.88; Anal. Calcd. for $\mathrm{C}_{23} \mathrm{H}_{16} \mathrm{ClN} \mathrm{C} \mathrm{80.81,} \mathrm{H} \mathrm{4.72,}$ $\mathrm{N}$ 4.10, Cl 10.37; Found C 80.73, H 4.61, N 4.07, Cl 10.30 . 
2.2c 4-(3-Methoxyphenyl)-2,6-diphenylpyridine $\left(\mathrm{C}_{24} \mathrm{H}_{19} \mathrm{NO} 4 \mathrm{c}\right)$ : White solid; m.p. $124-127^{\circ} \mathrm{C}(85 \%)$; $R_{\mathrm{f}}$ (petroleum ether:ethylacetate): 7:3(v/v) $=0.83 ; \mathrm{UV}$ $\left(\mathrm{CHCl}_{3}\right) \lambda_{\max }=308 \mathrm{~nm}$; FT-IR $(\mathrm{KBr})=3034,1596$, 1547, 1486, 1444, 1398, 1285, 1255, 1204, 1171, 1037, $872,775,692 \mathrm{~cm}^{-1} ;{ }^{1} \mathrm{H}$ NMR (400 MHz, DMSO- $\left.d_{6}\right) \delta$ 8.30-8.36 (d, 4H, $J=7.6 \mathrm{~Hz}, \mathrm{ArH}), 8.14$ (s, 2H, ArH), 7.50-7.59 (m, 8H, $J=7.6 \mathrm{~Hz}, \operatorname{ArH}), 7.45-7.48(\mathrm{~d}$, $1 \mathrm{H}, J=6.8 \mathrm{~Hz}, \mathrm{ArH}), 7.06-7.08(\mathrm{~d}, 1 \mathrm{H}, J=6.8 \mathrm{~Hz}$, $\mathrm{ArH}), 3.82\left(\mathrm{~s}, 3 \mathrm{H}, \mathrm{OCH}_{3}\right) ;{ }^{13} \mathrm{C}$ NMR $(100 \mathrm{MHz}$ DMSO- $\left.d_{6}\right): \delta 160.37,156.97,150.02,139.76,139.27$, 130.63, 129.67, 129.18, 127.45, 120.09, 117.2, 115.39, 113.22, 55.79; GCMS (EI): $m / z(\%) 337.15$ (100) $[\mathrm{M}]^{+}$; Anal. Calcd. for $\mathrm{C}_{24} \mathrm{H}_{19} \mathrm{NO}$ : C 85.43, H 5.68, N 4.15, O 4.74; found: C 85.51, H 5.59, N 4.25, O 4.65\%.

2.2d 4-(2-Fluorophenyl)-2,6-diphenylpyridine $\left(C_{23} H_{16} F N 4 d\right)$ : White solid; m.p. $119-120^{\circ} \mathrm{C}(88 \%)$; $R_{\mathrm{f}}$ (petroleum ether:ethylacetate): 7:3 $(\mathrm{v} / \mathrm{v})=0.83$; $\mathrm{UV}\left(\mathrm{CHCl}_{3}\right) \lambda_{\max }=260 \mathrm{~nm}$; FT-IR $(\mathrm{KBr})=3031$, 1591, 1544, 1490, 1451, 1395, 1288, 1209, 1204, 1114, 1074, 1025, 878, 759, $694 \mathrm{~cm}^{-1}$; ${ }^{1} \mathrm{H}$ NMR $(400 \mathrm{MHz}$, DMSO- $\left.d_{6}\right) \delta$ 8.32-8.36 (d, 4H, ArH), $8.10(\mathrm{~s}, 2 \mathrm{H}$, ArH), 7.83-7.87 (t, 1H, ArH), 7.45-7.48 (d, 1H, ArH), 7.06-7.085 (d, 1H, ArH); ${ }^{13} \mathrm{C}$ NMR (100 MHz DMSO$\left.d_{6}\right): \delta 160.37,156.97,150.02,139.76,139.27,130.63$, $129,67,129.18,127.45,120.09,117.2,115.39,113.22$, 55.79; GCMS (EI) $m / z(\%) 325.13(100)[\mathrm{M}]^{+}$; Anal. Calcd for $\mathrm{C}_{24} \mathrm{H}_{19} \mathrm{NO}$ : C 84.90, H 4.96, F 5.84, N 4.30; found: C 85.01, H 4.85, F 5.78, O 4.36\%.

2.2e 4-(5-Methylfuran-2-yl)-2,6-diphenylpyridine $\left(\mathrm{C}_{22} \mathrm{H}_{17} \mathrm{NO4e}\right)$ : Light brown solid; m.p. $162-164^{\circ} \mathrm{C}$ $(85 \%) ; R_{\mathrm{f}}$ (petroleum ether:ethylacetate): $7: 3(\mathrm{v} / \mathrm{v})=$ 0.83; UV $\left(\mathrm{CHCl}_{3}\right) \lambda_{\max }=260 \mathrm{~nm}$; FT-IR $(\mathrm{KBr})=$ $3058,2927,1591,1548,1495,1447,1394,1237$, $1122,1204,1114,1075,1027,878,758,691 \mathrm{~cm}^{-1}$; ${ }^{1} \mathrm{H}$ NMR $\left(400 \mathrm{MHz}, \mathrm{DMSO}-d_{6}\right) \delta 8.24-8.29(\mathrm{~d}, 4 \mathrm{H}$, ArH), 8.10 (s, 2H, ArH), 7.47-7.57 (m, 7H, ArH), 6.34 (s, 1H, ArH), 2.50 (s, 3H, ArH) ${ }^{13} \mathrm{C}$ NMR $(100 \mathrm{MHz}$ DMSO- $\left.d_{6}\right): \delta 155.30,151.40,146.10,136.20,129.30$, 127.60, 127.40, 118.60, 107.80, 101.80, 14.10; GCMS (EI) $\mathrm{m} / z$ (\%) $311.13(100)[\mathrm{M}]^{+}$; Anal. Calcd. for $\mathrm{C}_{24} \mathrm{H}_{19} \mathrm{NO}$ : C 84.86, H 5.50, N 4.50, O 5.14; found: C 84.91, H 5.45, N 4.54, O 5.10\%.

$2.2 \mathrm{f}$ 4-(Furan-2-yl)-2,6-diphenylpyridine $\left(\mathrm{C}_{21} \mathrm{H}_{15} \mathrm{NO}\right.$ 4f): Light brown solid; m.p. $168-170^{\circ} \mathrm{C}(88 \%) ; R_{\mathrm{f}}$ (petroleum ether:ethylacetate): 7:3 (v/v) $=0.82$; UV $\left(\mathrm{CHCl}_{3}\right) \lambda_{\max }=260 \mathrm{~nm}$; FT-IR $(\mathrm{KBr})=3058,1606$, 1541, 1487, 1454, 1414, 1244, 1158, 1073, 1010, 868,
$772,690 \mathrm{~cm}^{-1} ;{ }^{1} \mathrm{H}$ NMR $\left(400 \mathrm{MHz}, \mathrm{DMSO}-d_{6}\right) \delta 8.24$ 8.29 (d, 4H, ArH), 8.13-8.15 (s, 2H, ArH), 7.93-7.96 (s, 1H, ArH), 7.47-7.57 (m, 7H, ArH), 6.74-6.75 (d, $1 \mathrm{H}, J=1.6 \mathrm{~Hz}, \mathrm{ArH}) ;{ }^{13} \mathrm{C}$ NMR $(100 \mathrm{MHz}$ DMSO$\left.d_{6}\right): \delta 110.85,112.95,113.08,127.23,129.22,129.8$, 130.01, 139.55, 145.23, 151.43, 156.97; Anal. Calcd. for $\mathrm{C}_{21} \mathrm{H}_{15} \mathrm{NO}$ : C 84.82, H 5.08, N 4.71, O 5.38; Found C 84.86, H 5.01, N 4.77, O 5.42.

$2.2 \mathrm{~g}$ 2,6-Diphenyl-4-p-tolylpyridine $\left(\mathrm{C}_{24} \mathrm{H}_{19} \mathrm{~N} \quad \mathbf{4 g}\right)$ : White solid; m.p. $118-119^{\circ} \mathrm{C}(88 \%) ; R_{\mathrm{f}}$ (petroleum ether:ethylacetate): $7: 3(\mathrm{v} / \mathrm{v})=0.8 ; \mathrm{UV}\left(\mathrm{CHCl}_{3}\right) \lambda_{\max }=$ $304 \mathrm{~nm}$; FT-IR $(\mathrm{KBr})=3034,2936,1598,1548$, 1442, 1398, 1286, 1254, 1203, 1170, 1036, 871, 775, $691 \mathrm{~cm}^{-1} ;{ }^{1} \mathrm{H}$ NMR $\left(400 \mathrm{MHz}, \mathrm{DMSO}-d_{6}\right) \delta 8.29$ $8.3(\mathrm{~d}, 4 \mathrm{H}, J=7.2 \mathrm{~Hz}, \operatorname{ArH}), 8.14(\mathrm{~s}, 2 \mathrm{H}, \operatorname{ArH})$, $7.92-7.94(\mathrm{~d}, 2 \mathrm{H}, J=7.6 \mathrm{~Hz}, \operatorname{ArH}), 7.51-7.55(\mathrm{t}$, $4 \mathrm{H}, J=7.2 \mathrm{~Hz}, \mathrm{ArH}), 7.46-7.48(\mathrm{t}, 2 \mathrm{H}, J=7.2 \mathrm{~Hz}$, ArH), 7.34-7.36 (d, 2H, $J=7.6 \mathrm{~Hz}, \operatorname{ArH}), 2.48(\mathrm{~s}$, $\left.3 \mathrm{H}, \mathrm{CH}_{3}\right) ;{ }^{13} \mathrm{C}$ NMR $\left(100 \mathrm{MHz}\right.$ DMSO- $\left.d_{6}\right): \delta 156.96$, $149,139.41,135.18,130.14,129.63,129.17,127.54$, 127.38, 116.66, 21.25; Anal. Calcd. for $\mathrm{C}_{24} \mathrm{H}_{19} \mathrm{~N}$ : C 89.68, H 5.96, N 4.36; Found C 89.62, H 5.90, N 4.32.

2.2h 4-(4-Methoxyphenyl)-2,6-diphenylpyridine $\left(\mathrm{C}_{24} \mathrm{H}_{19} \mathrm{NO} 4 \boldsymbol{h}\right)$ : White solid; m.p. $98-100^{\circ} \mathrm{C}(80 \%)$; $R_{\mathrm{f}}$ (petroleum ether:ethylacetate): 7:3 (v/v) $=0.8 ; \mathrm{UV}$ $\left(\mathrm{CHCl}_{3}\right) \lambda_{\max }=301 \mathrm{~nm}$; FT-IR $(\mathrm{KBr})=3035,2936$, 1596, 1547, 1486, 1444, 1398, 1285, 1255, 1204, 1171, 1037, 750, $691 \mathrm{~cm}^{-1} ;{ }^{1} \mathrm{H}$ NMR $\left(400 \mathrm{MHz}\right.$, DMSO- $\left.d_{6}\right)$ $\delta$ 8.30-8.38 (d, 4H, $J=7.2 \mathrm{~Hz}, \operatorname{ArH}), 8.14(\mathrm{~s}, 2 \mathrm{H}$, ArH), 8.02-8.04 (d, 2H, $J=8 \mathrm{~Hz}, \mathrm{ArH}), 7.53-7.59$ (t, $4 \mathrm{H}, J=7.2 \mathrm{~Hz}, \mathrm{ArH}), 7.46-7.49(\mathrm{~d}, 2 \mathrm{H}, J=6.8 \mathrm{~Hz}$, ArH), 7.09-7.15 (d, 2H, J = 8 Hz, ArH), $3.82(\mathrm{~s}$, $\left.3 \mathrm{H}, \mathrm{CH}_{3}\right) ;{ }^{13} \mathrm{C}$ NMR $\left(100 \mathrm{MHz}\right.$ DMSO- $\left.d_{6}\right): \delta 160.83$, $156.90,149.54,139.40,130.40,130.27,129.61$, 129.11, 127.39, 116.38, 114.95, 55.80; Anal. Calcd. for $\mathrm{C}_{24} \mathrm{H}_{19} \mathrm{NO}$ : C 85.43, H 5.68, N 4.15, O 4.74; Found C 85.49, H 5.64, N 4.10, O 4.78.

2.2i 4-(2,6-Diphenylpyridin-4-yl)-N,N-dimethylbenzenamine $\left(\mathrm{C}_{25} \mathrm{H}_{22} \mathrm{~N}_{2} 4 \boldsymbol{4}\right)$ : Yellow solid; m.p. 137$139^{\circ} \mathrm{C}(86 \%) ; R_{\mathrm{f}}$ (petroleum ether:ethylacetate): $7: 3$ $(\mathrm{v} / \mathrm{v})=0.83 ; \mathrm{UV}\left(\mathrm{CHCl}_{3}\right) \lambda_{\max }=252 \mathrm{~nm} ; \mathrm{FT}-$ IR $(\mathrm{KBr})=3037,2936,1598,1525,1489,1442$, 1398, 1352, 1233, 1199, 1168, 1066, 1023, 818, 773, $695 \mathrm{~cm}^{-1} ;{ }^{1} \mathrm{H}$ NMR $\left(400 \mathrm{MHz}, \mathrm{DMSO}-d_{6}\right) \delta 8.27-8.30$ (d, 4H, $J=7.2 \mathrm{~Hz}, \mathrm{ArH}), 8.10$ (s, 2H, ArH), 7.90-7.93 (d, $2 \mathrm{H}, J=8.4 \mathrm{~Hz}, \mathrm{ArH}), 7.44-7.55(\mathrm{~m}, 6 \mathrm{H}, \mathrm{ArH})$, 6.82-6.84 (d, $2 \mathrm{H}, J=8.4 \mathrm{~Hz}, \mathrm{ArH}), 3.19\left(\mathrm{~s}, 3 \mathrm{H}, \mathrm{CH}_{3}\right)$; ${ }^{13} \mathrm{C}$ NMR $\left(100 \mathrm{MHz}\right.$ DMSO- $\left.d_{6}\right): \delta 155.20,152.00$, 
$150.10, \quad 136.20,129.30,128.30,127.60,127.40$, 118.00, 114.80, 40.20; Anal. Calcd. for $\mathrm{C}_{25} \mathrm{H}_{22} \mathrm{~N}_{2}$ : C 85.68, H 6.33, N 7.99; Found C 85.62, H 6.29, N 7.94.

$2.2 \mathrm{j}$ 2,6-Bis (4-chlorophenyl)-4-phenylpyridine $\left(C_{23}\right.$ $\left.\mathrm{H}_{15} \mathrm{Cl}_{2} \mathrm{NO} 4 \mathrm{j}\right)$ : White solid; m.p. $175-178^{\circ} \mathrm{C}(88 \%)$; $R_{\mathrm{f}}$ (petroleum ether:ethylacetate): $7: 3(\mathrm{v} / \mathrm{v})=0.83$; $\mathrm{UV}\left(\mathrm{CHCl}_{3}\right) \lambda_{\max }=252 \mathrm{~nm}$; FT-IR $(\mathrm{KBr})=3052$, 1598, 1544, 1490, 1449, 1413, 1384, 1239, 1174, 1091, 1012, 829, 761, $694 \mathrm{~cm}^{-1} ;{ }^{1} \mathrm{H}$ NMR (400 MHz, DMSO$\left.d_{6}\right) \delta$ 8.34-8.41 (d, 4H, ArH), $8.23(\mathrm{~s}, 2 \mathrm{H}, \mathrm{ArH})$, 8.03-8.06 (d, 2H, ArH), 7.50-7.61 (m, 7H, ArH); ${ }^{13} \mathrm{C}$ NMR $\left(100 \mathrm{MHz}\right.$ DMSO- $\left.d_{6}\right): \delta 155.75,150.31,137.86$, 134.65, 129.91, 129.55, 127.87, 117.31; Anal. Calcd. for $\mathrm{C}_{23} \mathrm{H}_{15} \mathrm{Cl}_{2} \mathrm{NO}$ : C 70.42, H 3.85, N 3.57, $\mathrm{Cl} 18.07, \mathrm{O}$ 4.08; Found C 70.36, H 3.80, N 3.51, Cl 18.01, O 4.04.

$2.2 \mathrm{k}$ 2,6-Bis (4-chlorophenyl)-4-(4-methoxyphenyl) pyridine $\left(\mathrm{C}_{24} \mathrm{H}_{17} \mathrm{NOCl}_{2} 4 \boldsymbol{k}\right)$ : White solid; m.p. 190 $191^{\circ} \mathrm{C}(88 \%) ; R_{\mathrm{f}}$ (petroleum ether:ethylacetate): $7: 3$ $(\mathrm{v} / \mathrm{v})=0.83 ; \mathrm{UV}\left(\mathrm{CHCl}_{3}\right) \lambda_{\max }=252 \mathrm{~nm} ; \mathrm{FT}-\mathrm{IR}$ $(\mathrm{KBr})=3052,2928,1602,1543,1512,1489,1426$, $1381,1291,1247,1177,1088,1011,824 \mathrm{~cm}^{-1} ;{ }^{1} \mathrm{H}$ NMR $\left(400 \mathrm{MHz}\right.$, DMSO- $\left.d_{6}\right) \delta 8.29-8.40(\mathrm{~d}, 4 \mathrm{H}$, $J=7.2 \mathrm{~Hz}, \mathrm{ArH}), 8.17(\mathrm{~s}, 2 \mathrm{H}, \mathrm{ArH}), 8.50-8.60(\mathrm{~d}$, $4 \mathrm{H}, J=7.2 \mathrm{~Hz}, \mathrm{ArH}), 7.45-7.50(\mathrm{~d}, 2 \mathrm{H}, J=6.8 \mathrm{~Hz}$, ArH), 7.09-7.12 (d, 2H, J = 8 Hz, ArH), $3.84(\mathrm{~s}$, $\left.3 \mathrm{H}, \mathrm{CH}_{3}\right) ;{ }^{13} \mathrm{C}$ NMR $\left(100 \mathrm{MHz}\right.$ DMSO- $\left.d_{6}\right): \delta 161.10$, $155.75,134.30,132.90,130.20,129.3,129,128.40$, 118, 114.80, 5.80; Anal. Calcd. for $\mathrm{C}_{24} \mathrm{H}_{17} \mathrm{NOCl}_{2}$ : C 70.95, H 4.22, N 3.45, O 3.94, Cl 17.45; Found C 70.91, H 4.25, N 3.41, O 3.97, Cl 17.40.

2.21 2,6-Bis (4-chlorophenyl)-4-(2-methoxyphenyl) pyridine $\left(\mathrm{C}_{24} \mathrm{H}_{17} \mathrm{Cl}_{2} \mathrm{NO} 4 \mathrm{ll}\right)$ : White solid; m.p. 164$166^{\circ} \mathrm{C}(86 \%) ; R_{\mathrm{f}}$ (petroleum ether:ethylacetate): 7:3 $(\mathrm{v} / \mathrm{v})=0.83 ; \mathrm{UV}\left(\mathrm{CHCl}_{3}\right) \lambda_{\max }=252 \mathrm{~nm} ;$ FT-IR $(\mathrm{KBr})=3049,2948,1601,1542,1512,491,1411$, $1383,1263,1245,1175,1126,1090,1014,827$, $749 \mathrm{~cm}^{-1} ;{ }^{1} \mathrm{H}$ NMR $\left(400 \mathrm{MHz}, \mathrm{DMSO}-d_{6}\right) \delta 8.22-8.32$ (d, $4 \mathrm{H}, J=8 \mathrm{~Hz}, \mathrm{ArH}), 8.04$ (s, 2H, ArH), 7.53-7.63 $(\mathrm{d}, 5 \mathrm{H}, J=8 \mathrm{~Hz}, \mathrm{ArH}), 7.43-7.45(\mathrm{t}, 1 \mathrm{H}, J=8 \mathrm{~Hz}$, ArH), 7.18-7.22 (d, 1H, $J=8 \mathrm{~Hz}, \mathrm{ArH}), 7.09-7.14$ (t, $J=8 \mathrm{~Hz}, 1 \mathrm{H}, \mathrm{ArH}), 3.84\left(\mathrm{~s}, 3 \mathrm{H}, \mathrm{CH}_{3}\right) ;{ }^{13} \mathrm{C} \mathrm{NMR}$ $\left(100 \mathrm{MHz}\right.$ DMSO- $\left.d_{6}\right): \delta \quad 161.10,155.75,134.30$, 132.90, 130.20, 129.3, 129, 128.40, 118, 114.80, 55.80. GCMS (EI) $m / z$ (\%): 405.07 (100) $[\mathrm{M}]^{+}$; Anal. Calcd. for $\mathrm{C}_{24} \mathrm{H}_{17} \mathrm{Cl}_{2} \mathrm{NO}$ : C 70.95, $\mathrm{H}$ 4.22, $\mathrm{Cl} 17.45, \mathrm{~N} 3.45$, O 3.94; found: C 71.03, H 4.18, Cl 17.36, N 3.51, O $3.92 \%$.
$2.2 \mathrm{~m}$ 2,6-Bis (4-chlorophenyl)-4-(3-methoxyphenyl) pyridine $\left(\mathrm{C}_{24} \mathrm{H}_{17} \mathrm{Cl}_{2} \mathrm{NO} 4 \mathrm{~m}\right)$ : White solid; m.p. 159 $160^{\circ} \mathrm{C}(80 \%) ; R_{\mathrm{f}}$ (petroleum ether:ethylacetate): $7: 3$ $(\mathrm{v} / \mathrm{v})=0.83 ; \mathrm{UV}\left(\mathrm{CHCl}_{3}\right) \lambda_{\max }=252 \mathrm{~nm} ; \mathrm{FT}-\mathrm{IR}$ $(\mathrm{KBr})=3062$, 2932, 1595, 1546, 1490, 1460, 1411, 1383, 1265, 1211, 1176, 1089, 1012, 833, $787 \mathrm{~cm}^{-1}$; ${ }^{1} \mathrm{H}$ NMR $\left(400 \mathrm{MHz}\right.$, DMSO- $\left.d_{6}\right) \delta 8.31-8.40(\mathrm{~d}, 4 \mathrm{H}$, $J=7.6 \mathrm{~Hz}, \operatorname{ArH}), 8.20(\mathrm{~s}, 2 \mathrm{H}, \mathrm{ArH}), 7.55-7.59(\mathrm{~d}$, $6 \mathrm{H}, J=7.6 \mathrm{~Hz}, \mathrm{ArH}), 7.42-7.50(\mathrm{~d}, 1 \mathrm{H}, J=6.8 \mathrm{~Hz}$, $\mathrm{ArH}), 7.05-7.12(\mathrm{~d}, 1 \mathrm{H}, J=6.8 \mathrm{~Hz}, \operatorname{ArH}), 3.88(\mathrm{~s}$, $\left.3 \mathrm{H}, \mathrm{CH}_{3}\right) ;{ }^{13} \mathrm{C}$ NMR $\left(100 \mathrm{MHz}\right.$ DMSO- $\left.d_{6}\right): \delta 161.20$, 155.20, 152, 138.90, 134.30, 132.90, 130.3, 129.3, 129, 119.7, 118, 114.80, 111.1, 55.80; GCMS (EI) $m / z(\%)$ 405.07 (100) $[\mathrm{M}]^{+}$; Anal. Calcd. for $\mathrm{C}_{24} \mathrm{H}_{17} \mathrm{Cl}_{2} \mathrm{NO}$ : C 70.95, H 4.22, Cl 17.45, N 3.45, O 3.94; found: C 71.03, $\mathrm{H} 4.18, \mathrm{Cl} 17.36, \mathrm{~N} 3.51$, O $3.92 \%$.

$2.2 \mathrm{n} \quad$ 2,6-Bis (4-chlorophenyl)-4-(2-fluorophenyl) pyridine $\left(\mathrm{C}_{23} \mathrm{H}_{14} \mathrm{Cl}_{2} \mathrm{FN} 4 \mathrm{n}\right)$ : White solid; m.p. 144$148^{\circ} \mathrm{C}(80 \%) ; R_{\mathrm{f}}$ (petroleum ether:ethylacetate): $7: 3$ $(\mathrm{v} / \mathrm{v})=0.83 ; \mathrm{UV}\left(\mathrm{CHCl}_{3}\right) \lambda_{\max }=252 \mathrm{~nm} ; \mathrm{FT}-\mathrm{IR}$ $(\mathrm{KBr})=3060,1603,1544,1490,1460,1411,1383$, $1265,1211,1176,1089,1012,826,750 \mathrm{~cm}^{-1} ;{ }^{1} \mathrm{H}$ NMR $\left(400 \mathrm{MHz}, \mathrm{DMSO}-d_{6}\right) \delta 8.25-8.31(\mathrm{~d}, 4 \mathrm{H}, J=8.4 \mathrm{~Hz}$, ArH), 8.20 (s, 2H, ArH), 7.8-7.85 (t, $1 \mathrm{H}, J=7.6 \mathrm{~Hz}$, ArH), 7.52-7.60 (d, 5H, $J=8.4 \mathrm{~Hz}, \mathrm{ArH}), 7.35-7.43$ $(\mathrm{t}, 2 \mathrm{H}, \mathrm{ArH}) ;{ }^{13} \mathrm{C}$ NMR $\left(100 \mathrm{MHz}\right.$ DMSO- $\left.d_{6}\right): \delta 116.0$, $118.0,124.9,129.0,129.3,130.90,132.8,134.3,152$, 155.2, 158.2; Anal. Calcd. for $\mathrm{C}_{23} \mathrm{H}_{14} \mathrm{Cl}_{2} \mathrm{FN}$ : C 70.07, $\mathrm{H} 3.58, \mathrm{Cl} 17.98, \mathrm{~F} 4.82, \mathrm{~N} 3.55$; found: C 70.10, H $3.52, \mathrm{Cl} 17.80, \mathrm{~F} 4.79, \mathrm{~N} 3.79 \%$.

$2.2 \mathrm{o}$ 2,6-Bis (4-chlorophenyl)-4-(furan-2-yl) pyridine $\left(\mathrm{C}_{21} \mathrm{H}_{13} \mathrm{Cl}_{2} \mathrm{NO}\right.$ 4o $)$ : White solid; m.p. $144-146^{\circ} \mathrm{C}$ $(87 \%) ; R_{\mathrm{f}}$ (petroleum ether:ethylacetate): $7: 3(\mathrm{v} / \mathrm{v})=$ 0.83; UV $\left(\mathrm{CHCl}_{3}\right) \lambda_{\max }=252 \mathrm{~nm}$; FT-IR $(\mathrm{KBr})=$ 3061, 1609, 1544, 1488, 1422, 1374, 1265, 1216, 1176, 1090, 1012, 826, $736 \mathrm{~cm}^{-1}$; ${ }^{1} \mathrm{H}$ NMR $(400 \mathrm{MHz}$, DMSO- $\left.d_{6}\right) \delta 8.27-8.38(\mathrm{~d}, 4 \mathrm{H}, J=8.4 \mathrm{~Hz}, \mathrm{ArH}), 8.21$ (s, 2H, ArH), 7.92-7.98 (s, 1H, ArH), 7.52-7.68 (d, $5 \mathrm{H}, J=7.6 \mathrm{~Hz}, \mathrm{ArH}), 6.72-6.8(\mathrm{~d}, 1 \mathrm{H}, J=4 \mathrm{~Hz}$, ArH) $;{ }^{13} \mathrm{C}$ NMR $\left(100 \mathrm{MHz}\right.$ DMSO- $\left.d_{6}\right): \delta 155.3,154$, 146.1, 142.9, 134.30, 132.90, 129.30, 129, 118, 107, 105; GCMS (EI) $m / z$ (\%) 365.04 (100) $[\mathrm{M}]^{+}$; Anal. Calcd. for $\mathrm{C}_{21} \mathrm{H}_{13} \mathrm{Cl}_{2} \mathrm{NO}$ : C 68.87, $\mathrm{H} 3.58, \mathrm{Cl} 19.36, \mathrm{~N}$ 3.82, O 4.37; found: C 69.05, H 3.54, Cl 19.39, N 3.75, O $4.27 \%$.

$2.2 \mathrm{p}$ 4-(2,6-Bis (4-chlorophenyl) pyridin-4-yl)-N,Ndimethylbenzenamine $\left(\mathrm{C}_{25} \mathrm{H}_{20} \mathrm{Cl}_{2} \mathrm{~N}_{2} 4 \mathrm{p}\right)$ : Yellow solid; m.p. $\quad 139-140^{\circ} \mathrm{C} \quad(79 \%) ; \quad R_{\mathrm{f}}$ (petroleum ether: 
ethylacetate): $7: 3(\mathrm{v} / \mathrm{v})=0.83 ; \mathrm{UV}\left(\mathrm{CHCl}_{3}\right) \lambda_{\max }=$ $252 \mathrm{~nm}$; FT-IR $(\mathrm{KBr})=3061,2932,1597,1527,1488$, 1432, 1359, 1265, 1189, 1201, 1090, 945, $814 \mathrm{~cm}^{-1}$; ${ }^{1} \mathrm{H}$ NMR $\left(400 \mathrm{MHz}\right.$, DMSO- $\left.d_{6}\right) \delta 8.31-8.35(\mathrm{~d}, 4 \mathrm{H}$, $J=8.4 \mathrm{~Hz}, \operatorname{ArH}), 8.12(\mathrm{~s}, 2 \mathrm{H}, \operatorname{ArH}), 7.89-7.95(\mathrm{~d}$, $2 \mathrm{H}, J=8.8 \mathrm{~Hz}, \mathrm{ArH}), 7.52-7.56(\mathrm{~d}, 4 \mathrm{H}, J=8.4 \mathrm{~Hz}$, ArH), 6.80-6.84 (d, 2H, $J=8.4 \mathrm{~Hz}, \mathrm{ArH}, 2.98(\mathrm{~s}, 6 \mathrm{H}$, $\left.2 \mathrm{CH}_{3}\right) ;{ }^{13} \mathrm{C}$ NMR $\left(100 \mathrm{MHz}\right.$ DMSO- $\left.d_{6}\right): \delta 155.2,152$, $150.1,134.3,132.90,129,128.3,127.4,118,114.8$, 40.2. GCMS (EI) $\mathrm{m} / z$ (\%) $418.10(100)[\mathrm{M}]^{+}$; Anal. Calcd. for $\mathrm{C}_{25} \mathrm{H}_{20} \mathrm{Cl}_{2} \mathrm{~N}_{2}$ : C 71.60, $\mathrm{H} 4.81, \mathrm{Cl} 16.91, \mathrm{~N}$ 6.68; found: C 71.55, H 4.78, Cl 16.98, N 6.69\%.

2.2q 4-(2-Chlorophenyl)-2,6-bis (4-chlorophenyl) pyridine $\left(\mathrm{C}_{23} \mathrm{H}_{14} \mathrm{Cl}_{3} \mathrm{~N} 4 \boldsymbol{q}\right)$ : White solid; m.p. $168-170^{\circ} \mathrm{C}$ (80\%); $R_{\mathrm{f}}$ (petroleum ether:ethylacetate): $7: 3(\mathrm{v} / \mathrm{v})=$ 0.83; UV $\left(\mathrm{CHCl}_{3}\right) \lambda_{\max }=252 \mathrm{~nm}$; FT-IR $(\mathrm{KBr})=$ 3034, 1603, 1544, 1490, 1413, 1383, 1263, 1174, 1090, $1012,824,751 \mathrm{~cm}^{-1} ;{ }^{1} \mathrm{H}$ NMR $\left(400 \mathrm{MHz}, \mathrm{DMSO}-d_{6}\right)$

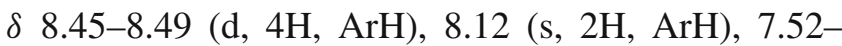
7.58 (d, 4H, ArH), 7.4-7.45 (d, 1H, ArH), 7.32-7.34 (d, 1H, ArH), 7.2-7.24 (t, 1H, ArH), $7.16(\mathrm{t}, 1 \mathrm{H}, \mathrm{ArH})$; ${ }^{13} \mathrm{C}$ NMR $\left(100 \mathrm{MHz}\right.$ DMSO- $\left.d_{6}\right): \delta 155.2,152,138.9$, 134.3, 132.90, 132.1, 130.7, 129.3, 129, 128.8, 127.4, 118; GCMS (EI) $m / z(\%) 409.02(100)[\mathrm{M}]^{+}$. Anal. Calcd. for $\mathrm{C}_{23} \mathrm{H}_{14} \mathrm{Cl}_{3} \mathrm{~N}$ : C 67.26, $\mathrm{H} 3.44, \mathrm{Cl} 25.90, \mathrm{~N}$ 3.41; found: C 67.31, H 3.39, Cl 25.87, N 3.43\%.

\section{Results and discussion}

Targeted compounds $\mathbf{4 a - q}$ were obtained starting from acetophenone $(2 \mathrm{mmol})$ and benzaldehyde $(1 \mathrm{mmol})$ in the presence of ammonium acetate $(4 \mathrm{mmol})$ under microwave conditions as a standard model reaction. Application of the prepared nanostructures $\left(\mathrm{MgAl}_{2} \mathrm{O}_{4}\right)$ as a catalyst was evaluated in this reaction. A wide variety of catalysts were employed to improve the yield of 2,4,6-triarylpyridine derivatives $\mathbf{4 a - q}$. As shown in Table 1, the best catalyst for this reaction is nanocrystalline $\mathrm{MgAl}_{2} \mathrm{O}_{4}$ which led to $95 \%$ yield of the desired product in $10 \mathrm{~min}$. We have started to use nanocrystalline $\mathrm{MgAl}_{2} \mathrm{O}_{4}$ as an efficient catalyst; since it has strong influence on the specific surface area and this character provides attraction between materials. Then, we have focused on optimizing conditions by using different amounts of $\mathrm{MgAl}_{2} \mathrm{O}_{4}$ nanocrystals. In the absence of catalyst, the reaction leads to a poor product yield and takes longer time for completion. The use of $5 \mathrm{~mol} \%$ of the catalyst produced maximum yield $(95 \%)$. Further amount of catalyst does not increase yield (Table 2).
Table 1. Effect of different catalysts on the reaction.

\begin{tabular}{|c|c|c|c|}
\hline Entry & Catalyst & Time (min) & Yield $(\%)$ \\
\hline 1 & $\mathrm{KOH}$ & 20 & 70 \\
\hline 2 & $\mathrm{NaOH}$ & 21 & 70 \\
\hline 3 & $\mathrm{~K}_{2} \mathrm{CO}_{3}$ & 32 & 60 \\
\hline 4 & $\mathrm{SbCl}_{3}$ & 18 & 68 \\
\hline 5 & $\mathrm{AlCl}_{3}$ & 20 & 62 \\
\hline 6 & $\mathrm{Al}_{2} \mathrm{O}_{3}$ & 42 & 50 \\
\hline 7 & $\mathrm{SbCl}_{3}-\mathrm{SiO}_{2}$ & 20 & 68 \\
\hline 8 & $\mathrm{MgO}$ & 30 & 60 \\
\hline 9 & $\mathrm{BaO}$ & 25 & 65 \\
\hline 10 & $\mathrm{NaOEt}$ & 22 & 70 \\
\hline 11 & L-Alanine & 25 & 65 \\
\hline 12 & Montmorillonite & 20 & 70 \\
\hline 13 & Nano crystalline $\mathrm{MgAl}_{2} \mathrm{O}_{4}$ & 10 & 95 \\
\hline
\end{tabular}

Table 2. Effect of amount of nanocrystalline $\mathrm{MgAl}_{2} \mathrm{O}_{4}$ in model reaction.

\begin{tabular}{lccc}
\hline Entry & Catalyst $(\mathrm{mol} \%)$ & Time $(\mathrm{min})$ & Yield $(\%)$ \\
\hline $\mathbf{1}$ & - & 40 & 15 \\
$\mathbf{2}$ & 2 & 20 & 67 \\
$\mathbf{3}$ & 3 & 15 & 79 \\
$\mathbf{4}$ & 5 & 10 & 95 \\
$\mathbf{5}$ & 7 & 10 & 95 \\
\hline
\end{tabular}

Table 3. Effect of different ammonia sources on the reaction.

\begin{tabular}{lccc}
\hline Entry & Ammonium source & Time (min) & Yield (\%) \\
\hline $\mathbf{1}$ & $\mathrm{NH}_{4} \mathrm{OAc}$ & 15 & 95 \\
$\mathbf{2}$ & $\left(\mathrm{NH}_{4}\right)_{2} \mathrm{CO}_{3}$ & 45 & 45 \\
$\mathbf{3}$ & $\mathrm{NH}_{4} \mathrm{~F}$ & 15 & 72 \\
$\mathbf{4}$ & $\mathrm{NH}_{2} \mathrm{CSNH}_{2}$ & 65 & 35 \\
$\mathbf{5}$ & $\mathrm{NH}_{2} \mathrm{COCH}_{3}$ & 70 & 64 \\
$\mathbf{6}$ & $\mathrm{NH}_{2} \mathrm{CONH}_{2}$ & 60 & 30 \\
\hline
\end{tabular}

Table 4. Optimization of reaction power for synthesis of compound 4a.

\begin{tabular}{lccc}
\hline Entry & $\mathrm{P}(\mathrm{w})$ & Time $(\mathrm{min})$ & Yield $(\%)$ \\
\hline $\mathbf{1}$ & 30 & 55 & Trace \\
$\mathbf{2}$ & 35 & 40 & 35 \\
$\mathbf{3}$ & 40 & 28 & 65 \\
$\mathbf{4}$ & 45 & 20 & 80 \\
$\mathbf{5}$ & 50 & 15 & 95 \\
$\mathbf{6}$ & 60 & 15 & 90 \\
\hline
\end{tabular}

Table 5. Reuse of catalyst in the model reaction.

\begin{tabular}{lccccc}
\hline Entry & $\mathbf{1}$ & $\mathbf{2}$ & $\mathbf{3}$ & $\mathbf{4}$ & $\mathbf{5}$ \\
Yield (\%) & 98 & 96 & 95 & 94 & 94 \\
\hline
\end{tabular}


Table 6. Solvent-free synthesis of 2,4,6-triarylpyridine derivatives catalysed by $\mathrm{MgAl}_{2} \mathrm{O}_{4}$ under microwave irradiation.

\begin{tabular}{|c|c|c|c|c|c|c|}
\hline \multirow[b]{2}{*}{ Entry } & \multirow[b]{2}{*}{$\mathrm{R}$} & \multirow[b]{2}{*}{$\mathrm{R}^{\prime}$} & \multirow[b]{2}{*}{ Product } & \multirow[b]{2}{*}{ Yield (\%) } & \multicolumn{2}{|c|}{ M.p. $\left({ }^{\circ} \mathrm{C}\right)$} \\
\hline & & & & & Found & Reported (lit.) \\
\hline 1 & $\mathrm{H}$ & $\mathrm{H}$ & $4 a$ & 95 & $135-137$ & $134-135^{49}$ \\
\hline 2 & $4-\mathrm{Cl}$ & $\mathrm{H}$ & $4 b$ & 89 & $127-128$ & $124-126^{20}$ \\
\hline 3 & 3-OMe & $\mathrm{H}$ & $4 c$ & 88 & $124-127$ & - \\
\hline 4 & $2-\mathrm{F}$ & $\mathrm{H}$ & $4 d$ & 91 & $119-120$ & - \\
\hline 5 & 5-Me-furan & $\mathrm{H}$ & $4 e$ & 89 & $162-164$ & - \\
\hline 6 & $\mathrm{C}_{4} \mathrm{H}_{3} \mathrm{O}$ (furan) & $\mathrm{H}$ & $4 f$ & 92 & $158-160$ & $168-170^{49}$ \\
\hline 7 & $4-\mathrm{Me}$ & $\mathrm{H}$ & $4 \mathrm{~g}$ & 84 & $119-120$ & $123-124^{20}$ \\
\hline 8 & 4-OMe & $\mathrm{H}$ & $4 \mathrm{~h}$ & 84 & 98-100 & $99-101^{20}$ \\
\hline 9 & $4-\mathrm{N}(\mathrm{Me})_{2}$ & $\mathrm{H}$ & $4 i$ & 87 & $137-139$ & $138-140^{49}$ \\
\hline 10 & $\mathrm{H}$ & $4-\mathrm{Cl}$ & $4 \mathbf{j}$ & 90 & $175-178$ & $188-190^{50}$ \\
\hline 11 & $4-\mathrm{OMe}$ & $4-\mathrm{Cl}$ & $4 k$ & 86 & 190-191 & - \\
\hline 12 & $2-\mathrm{OMe}$ & $4-\mathrm{Cl}$ & 41 & 82 & $164-166$ & - \\
\hline 13 & 3-OMe & $4-\mathrm{Cl}$ & $4 m$ & 80 & $159-160$ & - \\
\hline 14 & $2-\mathrm{F}$ & $4-\mathrm{Cl}$ & $4 n$ & 88 & $144-148$ & - \\
\hline 15 & $\mathrm{C}_{4} \mathrm{H}_{3} \mathrm{O}$ (furan) & $4-\mathrm{Cl}$ & 40 & 89 & $144-146$ & - \\
\hline 16 & $4-\mathrm{N}(\mathrm{Me})_{2}$ & $4-\mathrm{Cl}$ & $4 p$ & 81 & $139-140$ & - \\
\hline 17 & $2-\mathrm{Cl}$ & $4-\mathrm{Cl}$ & $4 q$ & 84 & $168-170$ & - \\
\hline
\end{tabular}

Then in determining the best ammonium source, we found that $\mathrm{NH}_{4} \mathrm{OAc}$ produced acetic acid in the reaction conditions that have good effect in progress of the reaction. Therefore, the experimental results convinced us that $\mathrm{NH}_{4} \mathrm{OAc}$ is the most effective ammonium source for this transformation. The reaction in the presence of other ammonium sources required longer reaction times, and produced lower yields (Table 3 ).

The effect of the reaction power was investigated by carrying out model reaction. It was shown that reaction at power of $50 \mathrm{w}$ was found to be optimal (Table 4). The reusability of the catalyst was also investigated. For this purpose, the same model reaction was again studied under optimized conditions. After completion of the reaction, by addition of hot ethanol, insoluble catalyst was filtered off. Then, catalyst was washed with acetone and dried. It was reused in subsequent reactions without reduction in activity as shown in Table 5. Results showed that nanocrystalline $\mathrm{MgAl}_{2} \mathrm{O}_{4}$ could be used for five successive runs without significant decrease in its catalytic activity. Finally, to evaluate the generality of this model reaction, we prepared a range of 2,4,6triarylpyridines under the optimized reaction conditions (Table 6).

Suggested mechanism is shown in Scheme 2. After Aldol condensation of aldehyde and acetophenone, second molecule of acetophenone is condensed by ammonia and forms enamine. The Michael addition of enamine on aldol product and then cyclization produces dihydropyridine. After that, oxidation takes place

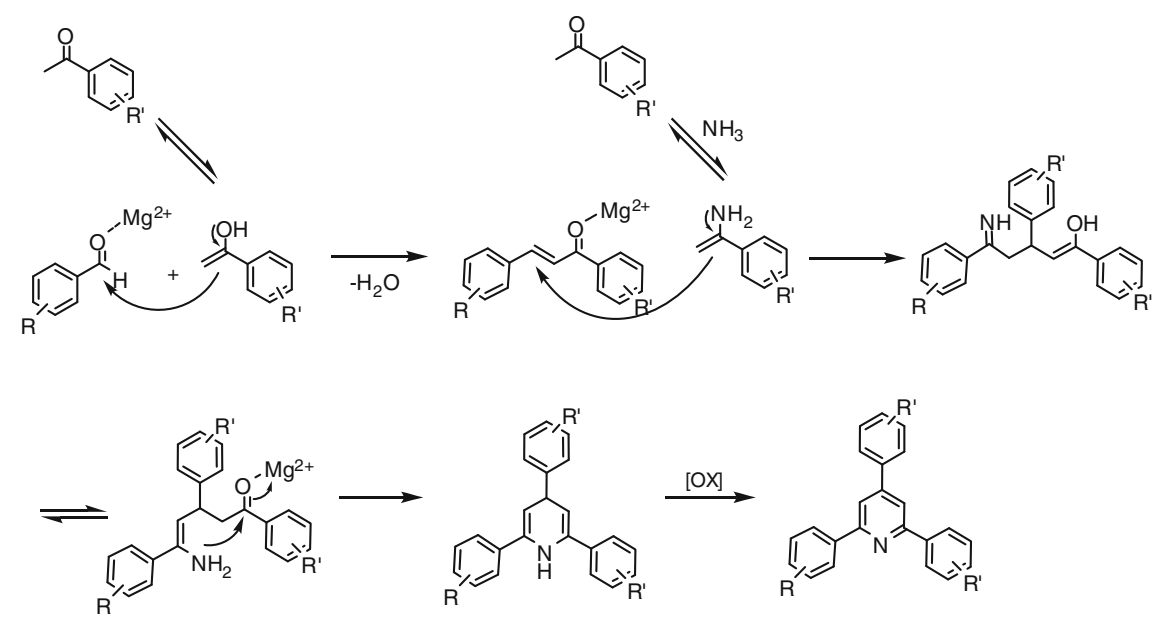

Scheme 2. Proposed mechanism of reaction. 
simply using the catalyst and affords the final product. Nanocrystalline $\mathrm{MgAl}_{2} \mathrm{O}_{4}$ facilitates all reaction routes such as Aldol condensation, Michael addition, cyclization and oxidation.

\section{Conclusion}

In summary, we have reported a one-pot and efficient method for the synthesis of 2,4,6-triarylpyridines using novel and applicable $\mathrm{MgAl}_{2} \mathrm{O}_{4}$ nanostructures. This protocol provides a very fast, good yield, low pollution, green and low cast procedure for the synthesis of these products. Moreover, the microwave-assisted combustion method is an economical and rapid method for the preparation of triarylpyridines with respect to energy, time and simplicity. We believe that this procedure appears to have an extensive scope with respect to variation in the pyridine 2- or 6- and 4-positions.

\section{Acknowledgement}

The authors gratefully acknowledge the financial support from the Research Council of the University of Kashan (Grant No. 256722/17), Iran.

\section{References}

1. De La Hoz A, Diaz-Ortiz A and Moreno A 2004 Curr. Org. Chem. 8903

2. Clark D E, Folz D C and West J K 2000 Microstruct. Proc. 287153

3. Bougrin K, Loupy A and Soufiaoui M 2005 J. Photochem. Photobiol. C6 139

4. Ju Y H and Varma R S 2005 Org. Lett. 72409

5. Kabalka G W, Wang L, Namboodiri V and Pagni R M 2000 Tetrahedron Lett. 415151

6. Kim B Y, Ahn J B, Lee H W, Kang S K, Lee J H, Shin J S, Ahn S K, Hong C I and Yoon S S 2004 Eur. J. Med. Chem. 39433

7. Enyedy I J, Sakamuri S, Zaman W A, Johnson K M and Wang S 2003 Bioorg. Med. Chem. Lett. 13(3) 513

8. Pillai A D, Rathod P D, Franklin P X, Patel M, Nivsarkar M, Vasu K K, Padh H and Sudarsanam V 2003 Biochem. Biophys. Res. Commun. 301(1) 183

9. Klimešová V, Svoboda M, Waisser K, Pour M and Kaustová J 1999 Il Farmaco 54(10) 666

10. Constable E C, Housecroft C E, Neuburger M, Phillips D, Raithby P R, Schofield E, Sparr E, Tocher D A, Zehnder M and Zimmermann Y 2000 J. Chem. Soc. Dalton Trans. (13) 2219

11. (a) Krohnke F and Zecher W 1962 Angew. Chem. Int. Ed. 1(12) 626; (b) Krohnke F 1976 Synthesis 1626

12. Zhao L X, Moon Y S, Basnet A, Kim E, Jahng Y, Park J G, Jeong T C, Cho W J, Choi S U, Lee C O, Lee S Y, Lee C S and Lee E S 2004 Bioorg. Med. Chem. Lett. 14 1333

13. Chubb F, Hay A S and Sandin R B 1953 J. Am. Chem. Soc. 75(23) 6042
14. Dilthey W 1921 J. Prakt. Chem. 102(8-10) 209

15. Lombard R and Stephen J P 1958 Bull. Soc. Chim. Fr. 1458

16. Zecher W and Krohnke F 1961 Chem. Ber. 94(3) 690

17. Frank R L and Seven R P 1949 J. Am. Chem. Soc. 71(8) 2629

18. (a) Potts K T, Cipullo M J, Ralli P and Theodoridis G 1981 J. Am. Chem. Soc. 103(12) 3584; (b) Potts K T, Cipullo M J, Ralli P and Theodoridis G 1981 J. Am. Chem. Soc. 103(12) 3585

19. Kobayashi T, Kakiuchi H and Kato H 1991 Bull. Chem. Soc. Jpn. 64(2) 392

20. Adib M, Tahermansouri H, Koloogani S A, Mohammadi B and Bi-janzadeh H R 2006 Tetrahedron Lett. 475957

21. Palacios F, de Retana A M O and Oyarzabal J 1996 Tetrahedron Lett. 37(26) 4577

22. Verma A K, Koul S, Pannu A P S and Razdan T K 2007 Tetrahedron 638715

23. Kumar A, Koul S, Razdan T K and Kapoor K K 2006 Tetrahedron Lett. 47837

24. Borthakur M, Dutta M, Gogoi S and Boruah R C 2008 Synlett (20) 3125

25. Cave G W V and Raston C L 2001 J. Chem. Soc., Perkin Trans. 13258

26. Zomordbakhsh S, Anaraki-Ardakani H, Zeeb M, Sadeghi M and Mazraeh-Seffid M 2012 J. Chem. Res. 36(3) 138

27. Cave G W V and Raston C L 2000 Chem. Commun. 2199

28. (a) Smith C B, Raston C L and Sobolev A N 2005 Green Chem. 7(9) 650; (b) Smith N M, Raston C L, Smith C B and Sobolev A N 2007 Green Chem. 9(11) 1185

29. Heravi M M, Bakhtiari Kh, Daroogheha $Z$ and Bamoharram F F 2007 Catal. Commun. 8(12) 1991

30. Davoodnia A, Bakavoli M, Moloudi R, TavakoliHoseini N and Khashi M 2010 Monatsh Chem. 141(8) 867

31. Nagarapu L, Peddiraju A R and Apuri S 2007 Catal. Commun. 8(12) 1973

32. Maleki B, Azarifar D, Veisi H, Hojati S F, Salehabadi H and Yami R N 2010 Chin. Chem. Lett. 21(11) 1346

33. Ren Y M and Cai C 2009 Monatsh. Chem. 140(1) 49

34. Montazeri N and Mahjoob S 2012 Chin. Chem. Lett. 23 419

35. Shinde P V, Labade V B, Gujar J B, Shingate B B and Shingare M S 2012 Tetrahedron Lett. 531523

36. Montazeri N, Ayoubi S F, Pourshamsian K and Bashtini F 2012 Oriental J. Chem. 28(1) 303

37. Reddy K S, Reddy R B, Mukkanti K, Thota G and Srinivasulu G 2011 Rasayan J. Chem. 4(2) 299

38. Davoodnia A, Razavi B and Tavakoli-Hoseini N 2012 E-J. Chem. 9(4) 2037

39. Maleki B, Salehabadi H, Sepehr Z and Kermanian M 2011 Collect. Czech. Chem. Commun. 761307

40. Mohammad Shafiee M R, Moloudi R and Ghashang M 2012 APCBEE Proc. 1221

41. Mohammad Shafiee M R and Moloudi R 2011 J. Chem. Res. 35(5) 294

42. Banerjee S and Sereda G 2009 Tetrahedron Lett. 50 6959 
43. Navaei Alvar E, Rezaei M, Navaei Alvar H, Feyzallahzadeh H and Yan Z F 2009 Chem. Eng. Commun. 196(11) 1417

44. Baudin C, Martinaz R and Pena P 1995 J. Am. Ceram. Soc. 78(7) 1857

45. Ganesh I, Bhattacharjee S, Saha B, Johnson R, Rajeshwari K, Sengupta R, Ramana Rao M and Mahajan Y 2002 Ceram. Int. 28(3) 245
46. Fairhurst C W 1992 Adv. Dent. Res. 678

47. Thomé L, Gentils A, Jagielski J, Garrido F and Thomé T 2007 Vacuum 81(10) 1264

48. Koroleva L 2004 Glass. Ceram. 61(9) 299

49. Huang X Q, Li H X, Wang J X and Jia X F 2005 Chin. Chem. Lett. 16(5) 607

50. Chiu C, Tang Z and Ellingboe J W 1999 J. Comb. Chem. 1(1) 73 